\title{
Navigated Three Dimensional Beta Probe for Optimal Cancer Resection
}

\author{
Thomas Wendler ${ }^{1}$, Joerg Traub ${ }^{1}$, Sibylle Ilse Ziegler ${ }^{2}$, and Nassir Navab ${ }^{1}$ \\ ${ }^{1}$ Chair of Computer Aided Medical Procedures (CAMP), TU Munich, Germany \\ twendler@ieee.org, \{traub, navab\}@cs.tum.edu \\ ${ }^{2}$ Nuclear Medicine Department, Klinikum rechts der Isar der Technische Universität \\ München, Germany \\ s-ziegler@lrz-muenchen.de
}

\begin{abstract}
In minimally invasive tumor resection, the desirable goal is to perform a minimal but complete removal of cancerous cells. In the last decades interventional nuclear medicine probes supported the detection of remaining tumor cells. However, scanning the patient with an intraoperative probe and applying the treatment are not done simultaneously. The main contribution of this work is to extend the one dimensional signal of a beta-probe to a four dimensional signal including the spatial information of the distal end of the probe. We generate a color encoded surface map of the scanned activity and guide any tracked surgical instrument back to the regions with measured activity. For navigation, we implement an augmented reality visualization that superimposes the acquired surface on a visual image of the real anatomy. Alternatively, a simulated beta-probe count rate in the tip of a tracked therapeutic instrument is simulated showing the count number and coding it as an acoustic signal. Preliminary tests were performed showing the feasibility of the new designed system and the viability of such a three dimensional intraoperative molecular imaging modality.
\end{abstract}

\section{Introduction}

A challenging task in tumor resection is the minimal but complete removal of malignant cells. In the ideal case, the surgeon performs an extirpation such that the security margins are maximized to avoid recurrence, while minimizing the impact on healthy tissue [1,2].

The standard procedure to confirm a complete removal is a pathological examination of the extracted tissue during the intervention. If the pathological examination detects cancerous cells at the margins, in the absence of spatial information, the surgeon can do nothing but enlarge the overall resection area.

Recently beta-probes have been introduced to improve the detection of remanent malignant tumor cells by means of nuclear labeling 3, 4. A cancer marker like FDG1, is injected into the patient before surgery. After the standard resection the surgeon scans the margins with the beta-probe. The detection of

$\overline{{ }^{118} F \text {-labeled }}$ 2-deoxy-2-fluoro-d-glucose, a positron-emitting glucose analogue and standard tracer for malignancy localization and staging [1]. 
unexpected high activity levels shows the occurrence of residual tumor tissue2. The physician has to memorize then the afflicted region, take away the probe, and apply the treatment with a surgical instrument at the place where the highest activity was measured.

The main contribution of this work is to improve the interventional use of beta-probes by combining it with a position and orientation tracking system to generate an activity encoded surface, and to use advanced visualization to represent the functional information to the surgeon. Thus, this work extends the one dimensional beta-probe device to a three dimensional imaging modality. In this paper we explain a prototypical system for recording the measured activity with its corresponding position, and either visualize it by superimposing the generated surface on an image of the real world or by simulating the beta-probe count rate at the tip of any tracked surgical instrument.

\section{Requirements and Design Considerations}

Hand held probes are commonly used as intraoperative diagnostic devices. They are capable of scanning arbitrary surfaces with a spatial accuracy only limited by the sensor size. Beta-probes are a specially interesting variant, since positrons (beta-plus-particles) penetrate tissue only up to $2[\mathrm{~mm}][3,5]$. This justifies the assumption that the activity measured is located at the surface next to the distal end of the probe and thus enables good spatial accuracy in detection.

Using a position localization system, e.g. an optical tracking system, we can acquire and synchronize recordings of spatial information and beta-particle counts. The combination of both data streams results in a three dimensional imaging device for arbitrary surfaces, which precisely memorizes the synchronized information of activity distribution, and position of the probe on the surface of the examined anatomy. A block diagram of a general navigated probe is shown in figure $1(\mathrm{a})$.

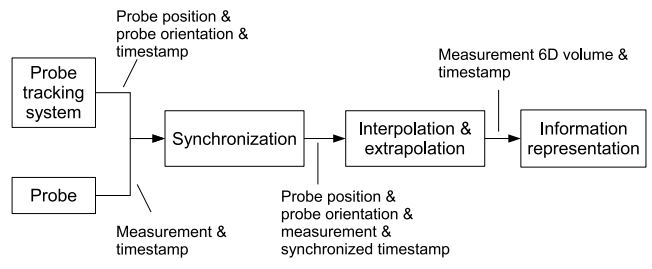

(a) Acquisition and synchronization.

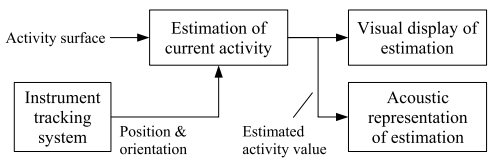

(b) Simulated probe.

Fig. 1. (a) The acquisition of position and orientation of the measurement device and the radioactive counts is recorded and synchronized, resulting in the generation of the three dimensional surface of the activity. (b) The presentation of the recorded activity at the tip of any tracked instrument is done either by displaying the count rate or using an acoustic signal

${ }^{2}$ Normally, the absolute value is not relevant, if it is high relative to the background. 
When designing a tracked beta-probe, we must consider the following issues:

1. Since the input is an asynchronous data flow, a proper synchronization system is required. The output is a synchronized four dimensional signal or even seven dimensional, when considering the orientation of the probe. Since we use the constraint that the probe will be moved only orthogonal to the surface, here we neglect the orientation. Furthermore, the detection of the positrons is rather insensitive to small changes in orientation compared to changes in distance 3 .

2. The data acquisition is not done on a uniformly spaced grid for position. Thus the surface map has different levels of details depending on the performed scan. One could use interpolation or extrapolation of the measurement in order to fill the missing data. This is optional, and has to be done with care and be subject to medical evaluation.

3. Beta-probes are not point sensors. They have usually a sensor disc with approximately $5[\mathrm{~mm}]$ radius. This has to be considered for the visualization. It also limits the detection accuracy to the sensor size. Nonetheless, multiple measurements within the sensor disc area can achieve a more precise detection of the radioactivity below the surface.

The information representation of the generated surface of synchronized data could be realized by visualizing the surface on a monitor superimposed on the real image of the surgery. This is especially useful for laparoscopic surgery since there is already a camera observing the region of interest that could be used to visualize the surface spatially aligned with the real anatomy (see figure 3(e)].

An alternative representation is the simulation of a real probe on the tip of a surgical instrument (figure 1(b)] . This could be implemented through an acoustic signal like a traditional radioactivity counter, by displaying the counts, or by a combination of both. Thus, for any instrument tip one could simulate the measurement of the probe using the previously acquired data. Since both are tracked in the same coordinate system no additional registration step is required.

\section{Implementation of a Three Dimensional Beta-Probe}

\subsection{Hardware Setup}

In our setup (figure 3(a) we used a beta-probe of IntraMedical Imaging LLC (Los Angeles, USA) with the NodeSeeker control unit. The sensitivity of the device with the used shielding is 4500 [counts per second $/ M B q]$ at $1[\mathrm{~cm}$ ] of a point-like source of ${ }^{18} \mathrm{~F}$. For tracking, we used an external optical infrared tracking system of ART GmbH (Weilheim, Germany) consisting of four ARTtrack2 cameras. The accuracy in localization for four cameras in a volume of $3 \times 3 \times 3\left[\mathrm{~m}^{3}\right]$ is $0.4[\mathrm{~mm}]$.

${ }^{3}$ From a geometric analysis, it can be shown that for angles smaller than $\pi / 20$ the relative error in the radioactive measurement lies below $15 \%$ for the dimensions of the probe used and a radioactivity source $2[\mathrm{~mm}]$ below. This error decreases with increasing distance to the source. 
We attached a 5DOF (degrees of freedom) tracking target directly on the axis of the probe as shown in figure 3(b) Instruments, cameras and the phantoms were also provided with infrared markers. The entire software for the navigation was implemented in $\mathrm{C}++$ and OpenGL within our research group.

\subsection{Synchronization of Data}

Depending on the information representation modality, two or three data streams need to be synchronized: tracking system, radioactivity measurement and (for the augmented reality visualization) the video stream. In order to do so, first the computer for the position tracking system and the NodeSeeker for radioactive counts are synchronized using NTP (network time protocol). The data acquired in the both computers is sent via UDP (user datagram protocol) including the time stamp of the moment of acquisition. In our software, we implemented the concept of a ring buffer and can thus correlate the data with the closest time stamps as implemented in [6]. The radioactive count measurements are acquired during a minimum of $100[\mathrm{~ms}]$ to get a statistically representative signa 4 . Since synchronization is the highest source of error in an augmented reality systems [7], we also synchronize the video and tracking data for a precise visualization. We use again a ring buffer and time stamp correlation.

\subsection{Calibration and Registration}

The distal end of the probe and tip of the instrument need to be calibrated. For this we acquire various poses of the attached tracking target moving the tip of the probe or instrument around a fixed point in space $p_{W}$. This leads to a set of equations $p_{W}=T_{M \rightarrow W}(t) p_{M}$, where $T_{M \rightarrow W}(t)$ is the transformation from the target coordinate system $M$ (marker) to the tracking coordinate system $W$ (world) at time $t, p_{W}$ is a constant point in the tracking coordinate system $W$, and $p_{M}$ the same constant point in the target coordinate system $M$. We obtain thus a linear equation system $A x=b$, where $A$ is a $3 n \times 6$ matrix for $n$ time samples, including the rotation part of $T_{M \rightarrow W}, b$ is a $3 n$ vector including the translation part of $T_{M \rightarrow W}$, and $x$ is the vector $\left(p_{t}^{T} p_{w}^{T}\right)^{T}$. Since both, probe and instrument, are tracked in the same coordinate system no further registration step is required to simulate the activity on the tip of the instrument.

For the visual representation, the surface is superimposed on the sequence of a video camera, which needs to be calibrated. For this we attach a $6 \mathrm{DOF}$ tracking target to it (see figure 3(b). With a chessboard pattern, we perform a fully automatic calibration in two steps. Firstly, we estimate the distortion coefficients and intrinsic camera parameters from a set of $n>10$ frames using a standard algorithm for camera calibration [8]. From this procedure the projection matrix $P$ to map three dimensional points onto the image plane is estimated.

\footnotetext{
${ }^{4}$ Assuming a Poisson distribution of the radioactive decay, it can be proved that for the estimator $\hat{\lambda}=N / \Delta t$ for the activity, the quotient standard deviation to expected value is $(\sqrt{\lambda} \Delta t)^{-1}$ with $\lambda$ as real activity, $N$ the number of counts and $\Delta t$ the observation window.
} 
In the second step, based on the same frames, we compute the rigid transformation $T_{C \rightarrow I}$ between the camera target coordinate system $C$ (camera) and one at the camera center $I$ (image). This is done by applying hand eye calibration 9] using corresponding poses of the camera in the chessboard coordinate system and poses of the tracking target in the tracking coordinate system $W$. Any known point in coordinate system $W$ can thus be projected into the image plane by $p_{2 D}=P T_{C \rightarrow I} T_{W \rightarrow C} p_{3 D}$, where $p_{2 D}$ is the point on the image plane, $p_{3 D}$ the point in the tracking coordinate system $W, P$ the projection matrix, $T_{C \rightarrow I}$ the rigid transformation, and $T_{W \rightarrow C}$ the transformation from coordinate systems $W$ and $C$, given by the tracking system.

\subsection{Information Representation}

Given the type of display, the $4 \mathrm{D}$ vector list (i.e. activity and $3 \mathrm{D}$ position) can be interpolated for the representation of the information. Depending on the representation, we use two different methods for interpolation/extrapolation.

Visual display using virtual or augmented reality: For the visual display, we use the standard Delaunay triangulation for a fast surface rendering over triangles. The nodes in 3D are the real positions of the beta-probe tip and their values are the real measurements. The color of the surface encodes the level of activity. Inside a triangle, the color is linearly interpolated using the distance from the point to be interpolated to the corners as weighting factors 5 . In case of a virtual reality visualization the generated surface is displayed using OpenGL. For the augmented reality, we use as the background texture the real camera image (e.g. of a tracked laparoscope) as in figure $3(\mathrm{e})$. The projection parameters in OpenGL are set to be the ones estimated during camera calibration. The camera center in the virtual scene is controlled by the pose data $T_{W \rightarrow C}$ provided by the tracking system and the calibrated rigid offset $T_{C \rightarrow I}$. The block diagram in figure 2 shows the work flow of the visual display for augmented and virtual reality modalities.

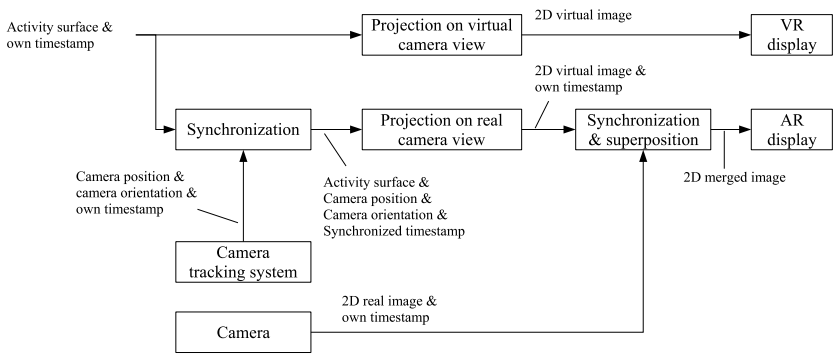

Fig. 2. Block diagram of the visual display using virtual and augmented reality

Simulated probe: The second method for representing the acquired information is to simulate the acquired data on the tip of any medical instrument e.g. a knife

${ }^{5}$ One can also visualize the point measurements without interpolation. This interpolation provides uniform activity surfaces, but has to be validated for medical use. 


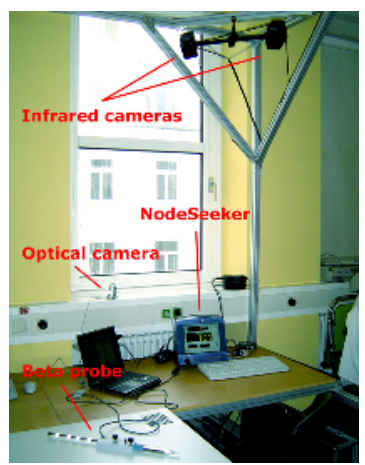

(a) Hardware setup.

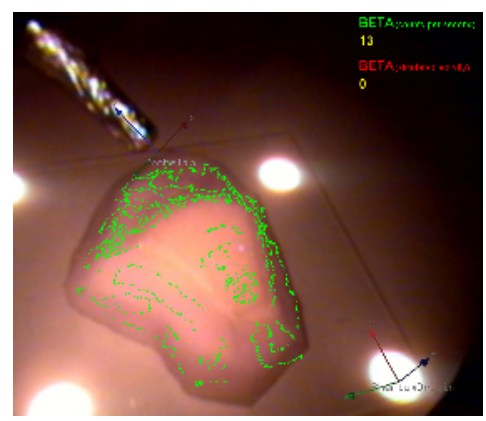

(c) Acquired points.

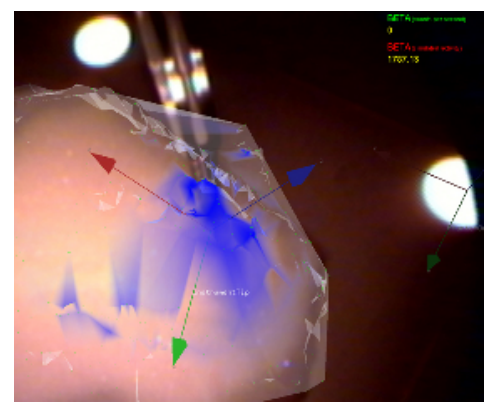

(e) Augmented reality view.

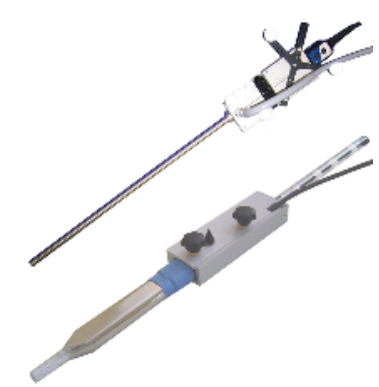

(b) Probe and laparoscope.

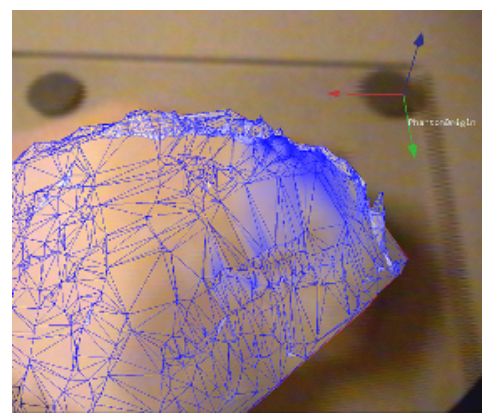

(d) Line mesh surface model.

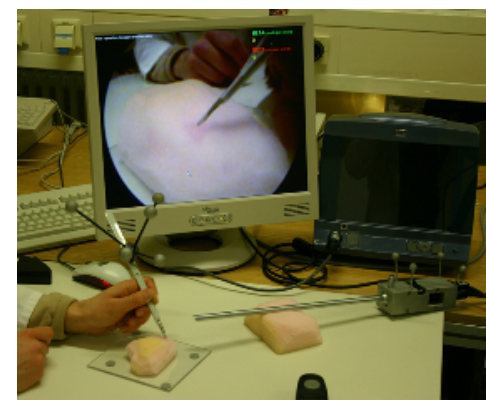

(f) Virtual probe.

Fig. 3. (a) The hardware setup consists of an optical tracking system (infrared cameras), a tracked camera and a beta-probe. (b) Laparoscopic camera and the extended beta-probe with marker targets for an external optical tracking system. (c)-(e) show screen shots of the system with the acquired data spatially registered into the view of the laparoscopic camera. Optionally all coordinate systems can be visualized during runtime to check the accuracy of the system. (e) visualizes the acquired activity. Blue is high measured activities. The upper number shows the measures of the real beta probe (which was turned off after acquisition), the lower number shows the simulated activity at the tip of the instrument. (f) is the same scene as (e) from outside showing the simulated activity with the 'NodeSeeker' turned off. 
for resection. Since we can not assume to have a measure at exactly the same location our instrument is positioned, we use radial basis weights to calculate the predicted activity ${ }^{5}$. The influence $I_{i}$ of a given point $i$ on the point to be interpolated is assumed to decay exponentially with the squared distance to the point $d_{i}$. We used $I_{i}=\exp \left\{-d_{i}^{2} /\left(2 \sigma^{2}\right)\right\}$ where $\sigma$ gives a radius of influence of each point in the interpolation. Additionally we defined a cut off radius. Finally the intrapolated/extrapolated measurement $\hat{M}$ was given as the weighted sum of the acquired measurements $M_{i}$, that is $\hat{M}=\left(\sum I_{i} M_{i}\right) /\left(\sum I_{i}\right)$.

Using the estimated measurement at the tip of the instrument, we either visualize the count rate on a monitor or represent it acoustically in form of a beep (like a traditional Geiger-Mueller counter), just like the one provided by the beta-probe during measurement, which gives extremely intuitive feedback. The codification used is PFM or PTM (pulse frequency or pulse time modulation) with a pulse width of $50[\mathrm{~ms}]$ and an acoustic signal of $800[\mathrm{~Hz}]$. The time between pulses is between 50 and $500[\mathrm{~ms}]$.

\section{Experiments and Results}

In order to validate the feasibility of the idea and estimate the accuracy of the system, a series of preliminary experiments were designed and performed. For this an ad-hoc three dimensional phantom is built. The selected prototype consists of a convex foam volume (e.g. simulating an organ) with attached infrared markers to allow acquisition of data relative to the coordinate system of the phantom and thus rigid motion of the phantom during acquisition and information representation. For the experiments, known volumes of an FDG solution of $2[\mathrm{MBq} / \mathrm{ml}]$ were injected to known positions of the phantom.

The experimental protocol consists in the acquisition of 1000 to 3000 points on a $10 \times 6 \times 4\left[\mathrm{~cm}^{3}\right]$ volume. The scan time for our phantom was between 2 and $5[\mathrm{~min}]$. However, the scanned degree of detail of the surface was higher than required for surgery, thus the acquisition time can be reduced to about one minute. The scan was performed with the probe approximately orthogonal to the surface of the phantom. In the visual information representation, points are displayed during the acquisition in order to get a visual feed-back of their distribution in space (figure $3(\mathrm{c})$. . The tip and origin of the probe, the instruments, as well as the origin of the phantom coordinate system can be optionally visualized in the camera view to validate the accuracy of the tracking system and the calibration of probe, instrument and camera during runtime. With the information acquired, the line and triangle meshes generated with the Delaunay algorithm are rendered and visualized superimposed onto the camera view (figure $3(\mathrm{~d})$. In case of the triangles, the color of the surface and its transparency are encoded as function of the activity measured.

The screen shots shown in figures $3(\mathrm{~d})$ and $3(\mathrm{e})$, as well as the multimedia additional materia 6 show the qualitative performance of the system. The visual registration of three dimensional surface and real surface was achieved up to

\footnotetext{
${ }^{6}$ Visit http://campar.in.tum.de/BetaProbe for a demo video.
} 
a satisfactory level $(1-2[\mathrm{~mm}])$. Moreover, the navigation system showed hot spots at the locations the radioactivity was injected and showed sufficient spatial accuracy for resection of residual tumor. In the simulated probe modality (figure $3(\mathrm{f})$, both the displayed count rate, as well as the acoustic signal, managed to guide the surgical instrument back to spots of high activity.

\section{Conclusions}

Beta-probes have been extensively studied and applied with success in medicine in the last decade. This is also the case for instrument tracking and advanced visualization in image guided surgery. In the present paper we introduce the integration of both technologies in a navigated beta-probe system for interventional use that benefits from both and allows improved minimal cancer resection. Furthermore, the concepts introduced represent one of the first approaches for three dimensional intraoperative molecular imaging.

The idea of tracking PET probes was mentioned in the past by several groups 10, 11, 12, but these patents and papers did not provide any theory or application. The combination with augmented reality and virtual measurement is new.

The experiments showed that despite the inherent sources of error in the system like statistical deviation, scattering, tracking inaccuracy, calibration of targets, tips and cameras, etc., the designed system is capable to perform well on the phantom model. In particular, the visual representation was appropriate for the targeted use showing the hot spots at their actual positions and with expected count rates. The simulated probe representation also guided the surgical instrument back to the regions with the highest measured activity.

As part of the future work, animal studies are scheduled. Eventhough no major considerations on deformability, motion and in general artifacts that arise from the clinical application were done, this work presents a new solution which enables the surgeons to perform a resection procedure, which is currently done with minimal support, by providing three dimensional intraoperative molecular imaging and thus by integrating functional information and surgical navigation.

Acknowledgements. The authors want to thank Dr. Markus Schwaiger and Dr. Farhad Daghighian for their support, as well as, Ben Mathison for his detailed technical support.

\section{References}

1. Phelps, M.E.: PET: The merging of biology and imaging into molecular imaging. The Journal of Nuclear Medicine 41(4) (2000) 661-681

2. Levin, C.S., Restom, T.S., Kapelanski, D.P., Hoh, C.K.: A simple surgical probe that directly detects positrons emitted from radiolabeled malignant tissue. Proceedings of the SNM 50th Annual Meeting 44(5) (2003) 
3. Daghighian, F., Mazziotta, J.C., Hoffman, E.J., Shenderov, P., Eshaghian, B., Siegel, S., Phelps, M.E.: Intraoperative beta probe: A device for detecting tissue labeled with positron or electron emitting isotopes during surgery. Med Phys 21(1) (1994) 153-157

4. Raylman, R.R., Wahl, R.L.: Evaluation of ion-implanted-silicon detectors for use in intraoperative positron-sensitive probes. Med Phys 23(11) (1996) 1889-1895

5. Weisenberg, A.G.: Gamma-ray blind beta probe. US Patent 6,317,622 (2001)

6. Sielhorst, T., Feuerstein, M., Traub, J., Kutter, O., Navab, N.: Campar: A software framework guaranteeing quality for medical augmented reality. In: Proceedings of CARS 2006 - to appear. (2006)

7. Hoff, W.A., Vincent, T.L.: Analysis of head pose accuracy in augmented reality. IEEE Trans. Visualization and Computer Graphics 6 (2000)

8. Hartley, R., Zisserman, A.: Multiple View Geometry in Computer Vision. 2nd edn. Cambridge University Press (2003)

9. Daniilidis, K.: Hand-eye calibration using dual quaternions. Journal of Robotics Research 18 (1999) 286298

10. Scibilia, G., Soluri, A.: Surgical probe for laparoscopy or intracavitary tumor localization. US patent 6,021,341 (2000)

11. Daghighian, F., Walker, R.E., Leon, B.J.: Methods and devices to expand applications of intraoperative radiation probes. US patent 6,510,336 B1 (2003)

12. Raylman, R.R., Hyder, A.: A dual surface barrier detector unit for beta-sensitive endoscopic probes. IEEE Trans. Nucl. Sci. 51(1) (2004) 117-122 